\title{
INFLUÊNCIA DA HIPERTENSÃO ARTERIAL NA INCIDÊNCIA DE DOENÇA RENAL TERMINAL EM NEGROS E MULATOS PORTADORES DE GLOMERULONEFRITE
}

RESUMO - OBjETIVO. Avaliar se a hipertensão arterial (HA) diagnosticada durante os primeiros dois meses de acompanhamento associa-se com a incidência de doença renal terminal (DRT) em negros e mulatos com glomerulonefrite, levando em consideração a idade, 0 sexo e 0 diagnóstico histológico.

Métodos. Estudo de coorte retrospectivo baseado em uma amostra de 120 pacientes negros e mulatos: $26 \mathrm{com}$ glomerulonefrite membranoproliferativa (GNMP), $58 \mathrm{com}$ glomeruloesclerose segmentar e focal (GSF) e 36 com outros tipos de glomerulonefrites (OTGN). HA foi considerada presente em pacientes usando anti-hipertensivos, em adultos apresentando média de três medidas de pressão arterial (PA) sistólica igual ou superior a 140 ou média das correspondentes medidas da PA diastólica igual ou superior a $90 \mathrm{mmHg}$. Para a faixa etária igual ou inferior a 18 anos foram utilizados os critérios da "Task Force on Blood Pressure in Children".

Resultados. HA no início do acompanhamento foi diagnosticada em 48 dos 120 pacientes $(41,6 \%)$. A incidência de
DRT foi aproximadamente 2,6 vezes maior em pacientes hipertensos (risco relativo $(\mathrm{RR})=2,62$; intervalo de confiança (IC) $95 \%=1,01-7,03 ; p=0,031)$. A associação entre $\mathrm{HA} \mathrm{e}$ aumento da incidência de DRT foi similar entre o grupo com idade menor ou igual a 18 anos e 0 grupo com idade superior a 18 anos. Mesmo após 0 ajuste para idade, sexo e tipo histológico, a incidência de DRT permaneceu maior (e marginalmente significante) em hipertensos que em normotensos (RR ajustado =2,15; IC $95 \%=0,86-5,39 ; p=0,07$ ).

Conclusōes. De acordo com os resultados, detecção precoce de HA em negros e mulatos com glomerulonefrite ajuda a identificar pacientes com maior risco de DRT, independente da idade, do sexo e do tipo histológico. Outros trabalhos são necessários para determinar em que extensão a severidade e grau de controle da HA contribuem para o desenvolvimento de DRT em negros e mulatos com glomerulonefrite.

UnITERMOS: Hipertensão arterial. Doença renal terminal. Glomerulonefrite. Raça. Negro. Mulato.

\section{INTRODUÇÃO}

Em um estudo prévio realizado na Bahia em pacientes com diagnóstico de glomerulonefrites comprovado através de biopsia renal foi mostrado, entre os hipertensos, uma tendência para um risco maior de Doença Renal Terminal (DRT) em pessoas classificadas como negras ou mulatas do que em brancos'. Este

*Correspondência

Rua Mar. Floriano, 448 - apt. 1301 - Canela

Salvador - BA - CEP:40110-010

E-mail: aaslopes@ufba.br achado em pacientes com glomerulonefrite é consistente com o que tem sido relatado em pacientes com hipertensão arterial $(\mathrm{HA})$ primária $^{2}$, sugerindo que na presença de elevação da pressão arterial a perda funcional renal é mais rápida no negro do que no branco. Formas mais graves de hipertensão e/ou uma maior susceptibilidade dos negros para lesão renal na presença de pressão arterial elevada pode ser uma das razões para a maior incidência de DRT em negros do que em brancos, particularmente DRT secundária à hipertensão arterial, ao dia- betes e às glomerulonefrites ${ }^{3-5}$. Além da raça e da presença de $\mathrm{HA}$, a idade e $\mathrm{O}$ sexo do paciente interferem com a evolução da doença renal6,7. Em pacientes com glomerulonefrites, o tipo histológico tem sido também relacionado com a presença de insuficiência renal à época da biópsia renal e com a progressão para DRT ${ }^{8-10}$. Para o presente estudo se utilizou uma subamostra de um estudo prévio'. A análise se restringiu a pacientes negros e mulatos portadores de glomerulonefrites com o objetivo de avaliar se hipertensão arterial identificada no início 
do acompanhamento nefrológico está associada com uma maior incidência de DRT em pessoas destes grupos raciais, ao se levar em consideração o tipo histológico, o sexo e a idade na época da consulta nefrológica.

\section{Métodos}

Trata-se de um estudo de coorte retrospectivo utilizando dados de pacientes acompanhados no Hospital Universitário Prof. Edgard Santos (HUPES), da Universidade Federal da Bahia (UFBA), entre 1970 e 1996. Neste período, 966 pacientes tiveram laudos de biópsias renais arquivados no Serviço de Anatomia Patológica do HUPES. Foram selecionados pacientes negros e mulatos com pelo menos seis meses de acompanhamento e níveis iniciais de creatinina sérica inferior a 4,0 mg/dl. Não foram incluídos pacientes com doenças do colágeno, no sentido de manter a amostra uniforme e reduzir a possibilidade da influência do uso crônico de corticosteróides na elevação dos níveis de pressão arterial. Portadores de glomerulo-nefrites crescênticas foram excluídos, visto que muitos já se apresentam com insuficiência renal avançada. Pacientes com glomerulonefrite aguda e lesão mínima não foram selecionados considerando o risco baixo de evolução para DRT.

Os critérios do estudo foram preenchidos por 120 pacientes, sendo 26 portadores de glomerulonefrite membranoproliferativa (GNMP), 58 de glomeruloesclerose segmentar e focal (GSF) de 36 de outros tipos de glomerulonefrite (OTGN). O grupo OTGN foi formado por 33 pacientes com formas proliferativas e três com glome-rulonefrite membranosa. Pacientes usando drogas antihipertensivas (excluindo diuréticos na vi- gência de síndrome nefrótica) foram classificados como hipertensos. Na ausência de anti-hipertensivos, pacientes com idade $>18$ anos foram considerados hipertensos quando apresentaram, nos dois primeiros meses de acompanhamento, uma média $\geq 140 \mathrm{~mm} \mathrm{Hg}$ nas três primeiras medidas da pressão arterial sistólica ou $\geq 90 \mathrm{~mm} \mathrm{Hg}$ nas medidas da pressão arterial diastólica. Para indivíduos com idades $\leq 18$ anos foram usados os critérios recomendados pela Task Force on Blood Pressure in Children5". Idade ( $\leq 18 \mathrm{e}>$ 18 anos), sexo, HA, tipo histológico (GNMP $(n=50)$, EGF $(n=80)$ e OTGN $(n=49))$ foram tratados como potenciais confundidoras ou modificadoras de efeito da associação entre raça e incidência de DRT.

\section{Análise Estatística}

As comparações de médias e de proporções foram feitas através do teste $t$ para amostras independentes $\mathrm{e}$ do teste do qui-quadrado, respectivamente, utilizando-se procedimentos do "Statistical Package for the Social Sciences", SPSS'12. Ajustes multi-variados da associação entre HA e DRT foram feitos através de modelos de risco proporcional de $\mathrm{Cox}^{13}$. O tempo de seguimento foi definido como o intervalo entre a data da primeira consulta e data do diagnóstico de DRT (ou data da última consulta em pacientes que não desenvolveram DRT). Idade $(\leq 18$ e $>18$ anos), sexo e tipo histológico foram tratados como variáveis potencialmente confundidoras. Foram incluídas duas variáveis indicadoras de tipo histológico: GNMP e OTGN. GSF foi utilizada como referência. Avaliou-se se a associação entre hipertensão arterial e DRT era modificada pela idade. Nos modelos de Cox, a presença de modificação de efeito (i.e., interação estatística) foi avaliada através do coeficiente do produto entre duas co-variáveis. Foram considerados significantes valores de $p$ menores do que $5 \%$, para testes estatísticos bi-caudais.

\section{Resultados}

Na Tabela I, as características dos pacientes são descritas de acordo com a presença $(n=48)$ ou ausência de HA $(n=72)$. A média $(28,2 \pm \mid 4,7$ versus $19,7 \pm||, 8$ anos; $p=0,001$ ) e mediana ( 27,9 versus 17,0 anos) de idade foram maiores no grupo de hipertensos. 0 percentual de pessoas com idade $>18$ anos foi significantemente maior $(p<0,00 \mathrm{I})$ em hipertensos que em normotensos ( $81,3 \%$ versus $45,8 \%)$. Houve um predomínio de pessoas do sexo masculino na amostra, sendo, no entanto, semelhante entre hipertensos $(60,4 \%)$ e normotensos $(59,7 \%)$. Em hipertensos, observou-se distribuição similar dos tipos histológicos. Contrariamente em normotensos, a distribuição não foi uniforme, havendo um menor percentual $(13,9 \%)$ de pessoas com GNMP e um maior percentual de portadores de GSF (58,3\%). HA esteve presente em 16 dos 26 pacientes $(61,5 \%)$ com GNMP, em 16 dos 58 $(27,6 \%)$ com GSF e em 16 dos 36 (44\%) com OTGN.

A Tabela 2 mostra que a incidência de DRT foi significantemente maior ( $R R=2,62 ; \quad$ IC $\quad 95 \%=1,01-7,03$; $p=0,03 \mathrm{I}$ ) em hiper-tensos (incidência $=43,0 / 10^{4}$ pessoas-mês) do que em normotensos (incidência $=16,4 / 10^{4}$ pessoas-mês), tanto no grupo com idade menor ou igual a 18 ( $R R=3,70$; IC $95 \%=0,49-27,37)$ quanto no grupo com idade superior a 18 anos ( $R R=2$, I2; IC 95\%=0,67-7,24). De 


\section{Tabela 1 - Características dos pacientes de acordo com a presença e ausência de hipertensão arterial}

\begin{tabular}{|c|c|c|c|}
\hline \multirow[b]{3}{*}{ Idade } & \multicolumn{2}{|c|}{ Hipertensão } & \multirow[b]{2}{*}{ valor } \\
\hline & Sim & Não & \\
\hline & $N=48$ & $N=72$ & dep \\
\hline média $\pm D P$ & $28,2 \pm 14,7$ & $19,7 \pm 11,8$ & 0,001 \\
\hline mediana & 27,9 & 17,0 & \\
\hline$\%>18 \operatorname{anos}(n / N)$ & $81,3(39 / 48)$ & $45,8(33 / 72)$ & $<0,00 \mid$ \\
\hline \multicolumn{4}{|l|}{ Sexo } \\
\hline$\%$ Masculino (n/N) & $60,4(29 / 48)$ & $59,7(43 / 72)$ & 0,939 \\
\hline \multicolumn{4}{|l|}{ Tipo Histológico* } \\
\hline \% GNMP (n/M) & $33,3(16 / 48)$ & $13,9(10 / 72)$ & 0,011 \\
\hline$\%$ GSF $(n / N)$ & $33,3(16 / 48)$ & $58,3(42 / 72)$ & \\
\hline$\% \operatorname{OTGN}(n / N)$ & $33,3(16 / 48)$ & $27,8(20 / 72)$ & \\
\hline
\end{tabular}

* GNMP = glomerulonefrite membranoproliferativa,

GSF = glomeruloesclerose segmentar e focal,

OTGN = outras glomerulonefrites

Tabela 2 - Comparação das incidências de doença renal terminal entre hipertensos e normotensos no grupo geral e estratificada pela idade geral

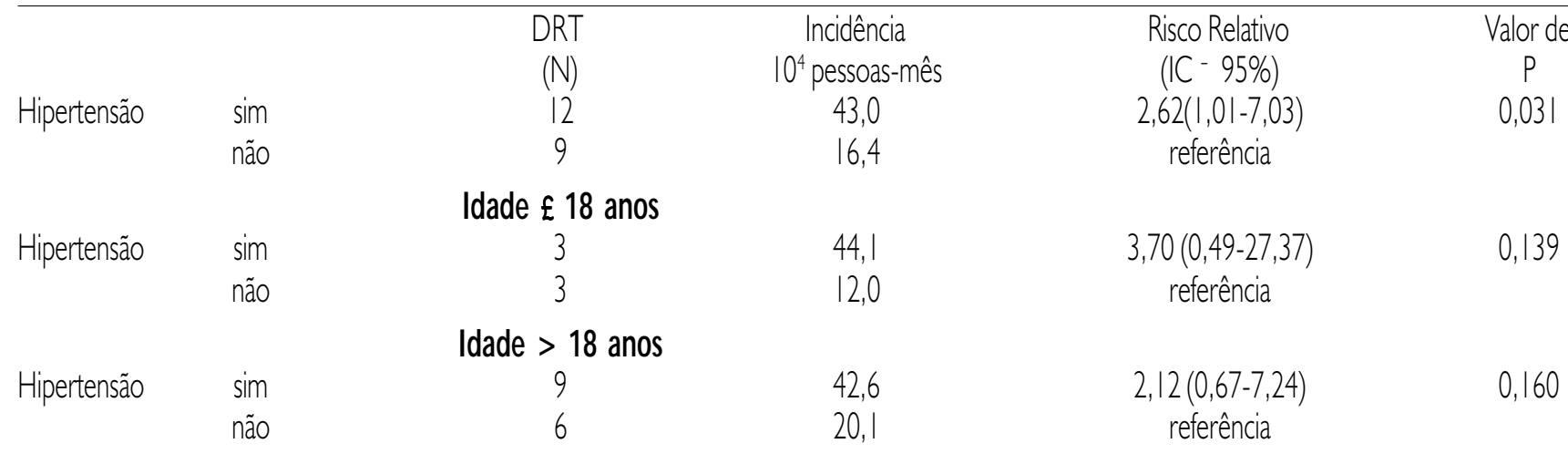

Valor de $\mathbf{P}$ do teste de heterogeneidade no RR entre os níveis da variável idade $=0,572$

DRT = Doença Renal Terminal

${ }^{\uparrow} \mathrm{IC}=$ Intervalo de Confiança

acordo com o teste de homogeneidade, a estimativa do RR não foi significantemente modificada pela idade $(p=0,572)$. Na análise através regressão de Cox (Tabela 3), a incidência de DRT foi também significante $(p=0,03 \mathrm{I})$ maior em hipertensos $(R R=2,63 ; I C$ $95 \%=1,09-6,30)$. Esta associação não se alterou e permaneceu estatistica- mente significante após o ajuste para idade e sexo $(R R=2,50 ; \quad I C$ $95 \%=1,02-6,13 ; p=0,046)$. A associação entre hipertensão arterial e maior risco de DRT ficou próxima ao nível de significância estatística $(R R=2,15 ; \quad I C$ $95 \%=0,86-5,39 ; \quad p=0,07), \quad$ mesmo após ajustar para todas as covariáveis (i.e., idade, sexo e tipo histológico).

\section{Discussão}

De acordo com os resultados do presente estudo, em pacientes negros e mulatos do estado da Bahia com diagnóstico de glomerulonefrite à biopsia renal, a presença de hipertensão no início do acompanhamento nefrológico é fator de risco para DRT, independente da idade, do sexo e do 


\section{Tabela 3 - Riscos relativos da associação entre hipertensão arterial doença renal terminal, não ajustado e ajustado através regressão de Cox}

\begin{tabular}{lccc}
\hline Modelos & Risco Relativo & IC $95 \%$ & Valor de $p$ \\
Não Ajustado & 2,63 & $1,09-6,30$ & 0,031 \\
Ajustado para Idade e Sexo & 2,50 & $1,02-6,13$ & 0,046 \\
Ajustado para Idade, Sexo e Tipo Histológico & 2,15 & $0,86-5,39$ & 0,07 \\
IC = Intervalo de Confiança & & & \\
\hline
\end{tabular}

tipo histológico. É importante notar que, em um estudo prévio, foi mostrada evidência de que os negros e mulatos têm um risco maior de DRT do que os brancos, apenas no grupo de hipertensos'. Os resultados foram interpretados como condizentes com a evidência de que HA é mais grave e mais relacionada com lesão renal em negros do que em brancos ${ }^{2,14,15}$. Maior influência de HA na progressão da insuficiência renal em negros do que em brancos tem sido também observada em pacientes submetidos a transplante renal ${ }^{16}$. Expressão aumentada para fatores de crescimento, particularmente TGF-beta, tem sido apontada como uma possível explicação para a predisposição do negro para formas mais graves de hipertensão arterial e maior incidência de DRT ${ }^{17-19}$.

O ajuste para tipo histológico realizado no presente trabalho contempla o achado de que HA se associa com tipo de nefropatia ${ }^{20}$. No presente estudo, a prevalência de HA foi maior nos pacientes com GNMP do que nos pacientes com outros tipos histológicos. $\mathrm{O}$ ajuste para o tipo histológico levou também em consideração um estudo prévio que sugeriu um maior risco de DRT entre os portadores de GNMP comparado com os portadores de outros tipos de glomerulonefrites'. Além do mais, tem sido mostrado diferenças na distribuição de tipos de glomerulonefrites entre negros e brancos, parecendo haver maior predisposição dos negros para GSF2-23. De acordo com os resultados do presente estudo, no entanto, o tipo histológico não explica a maior incidência de DRT em pacientes com glomerulonefrites portadores de $\mathrm{HA}$.

Embora os resultados observados nesta amostra de mulatos e negros do estado da Bahia estejam condizentes com investigações prévias, não se pode negar as limitações de um estudo retrospectivo. A seleçãao de um número limitado de variáveis com pequena probabilidade de erro de classificação deve ter, no entanto, reduzido a influência de viés de informação. As análises estratificadas por faixas etárias foram realizadas no sentido de contemplar os diferentes critérios para classificar HA entre os adultos e os pacientes mais jovens. É importante notar que a associação entre HA e DRT não foi significantemente diferente entre o grupo com idade $\leq 18$ e o grupo $>18$ anos. Os dados sugerem que nos dois grupos etários a incidência de DRT é maior em negros e mulatos do que em brancos portadores de HA. Embora o tempo de acompanhamento até o desenvolvimento do evento ou até a última data em que o paciente foi avaliado no serviço tenha sido levado em consideração na análise, a influência da perda de seguimento nos resultados do presente estudo não pode ser definitivamente afastada.

Infelizmente não foi possível avaliar se a incidência de DRT foi influenciada pela intensidade da redução da pressão arterial, pelo grau de controle da pressão arterial durante o dia e pelo tipo de anti-hipertensivo utilizado. Estas questões são, em verdade, dificeis de serem avaliadas em estudos retrospectivos. Um estudo de coorte prospectivo mostrou evidência de que o nível de pressão arterial registrado pela manhã antes da utilização de anti-hipertensivos é um indicador do grau de controle durante o dia e um preditor do declínio da filtração glomerular24. $\mathrm{O}$ estudo Modification of Diet in Renal Disease
(MDRD) forneceu dados permitindo estimar a influência do controle da HA sobre a função renal em negros e brancos portadores de nefropatias em geral, portanto, não restrito a pacientes com glomerulonefrites ${ }^{25}$. De acordo com os resultados do MDRD, redução da pressão arterial média (PAM) para um nível menor ou igual a $92 \mathrm{~mm} \mathrm{Hg}$ lentifica a progressão da insuficiência renal. Além do mais, o benefício de um controle mais rigoroso da HA foi mais notório nos negros do que nos brancos. O ensaio clínico em andamento, African American Study of Kidney Disease and Hypertension (AASK), além de testar o benefício em se reduzir mais intensamente a PAM, compara os efeitos de diferentes classes de anti-hipertensivos na proteção renal em negros ${ }^{26}$. Esperamos que os resultados do AASK sirvam para orientar a meIhor forma de tratar hipertensão arterial no sentido de reduzir a progressão da insuficiência renal e de prevenir DRT, particularmente em negros.

Em conclusão, os dados do presente estudo sugerem que, independente do sexo, da idade e do tipo histológico de glomerulonefrite, HA identificada no início do acompanhamento nefrológico é um fator de risco para DRT em pacientes negros e mulatos com glomerulonefrites. É importante avaliar a influência da gravidade da HA e do controle adequado da pressão arterial em reduzir a incidência de DRT nestes pacientes. Devem também ser consideradas as interações da hipertensão arterial com outros fatores relacionados com o risco de DRT, como a intensidade da proteinúria e a taxa inicial de filtração glomerular. 


\section{SUMmarY}

\section{INFLUENCE OF HYPERTENSION ON THE INCIDENCE OF END-STAGE RENAL DISEASE IN NEGROES AND MULATTOES WITH GLOMERU- LONEPHRITIS}

OBIECTNE. Toassess whether hypertension diagnosed duringthe first two monthsoffollowup is associated with the incidence of end-stage renal disease (ESRD) in negroes and mulattoes with glomerulonephritisaftertakinginto account age, gender and the histological diagnosis.

METHODS. A retrospective cohort study based on a sample of I 20 negroand mulatto patients with glomerulonephritis, 26 with membranoproliferative glomerulonephritis (MPGN), 58 with focal segmental glomerulosclerosis (FSG) and 36 with other types of glomerulonephritis (OTGN). Hypertension was considered present if antihypertensive treatment was used, in adults (age $>18$ years) with a mean of the three first systolic blood pressures of $140 \mathrm{mmHg}$ or a mean of the corresponding diastolic blood pressure of $90 \mathrm{~mm} \mathrm{Hg}$ or greater. For ages equal to or below 18 years the criteria recommended by the "Task Force on Blood Pressure in Children"were used.

RESULTS. Hypertension was diagnosed in 48 of the 120 patients (41.6\%). The incidence of ESRD was approximately $2.6 \mathrm{ti}$ meshigherin hypertensive patients (relative $\operatorname{risk}(R R)=2.62 ; 95 \%$ confidence interval $(C l)=1.01-7.03, p=0.031$ ). This association between hypertension and higher risk of ESRD was similar between the group younger than 19 and the group older than 18 years. Even after adjusting for age, gender and histologic type, the incidence of ESRD remained higher (and marginally significant) in the hypertensive than in the normotensive group (adjusted $R R=2.15 ; 1 C$ $95 \%=0.86-5.39, p=0.07)$.

CONCLUSIONs. According to the findings, early detection of hypertension in negroes and mulattoes with glomerulonephritis helps to identify patients with higher risk of ESRD, independently of age, gender and histologic type. Further research is needed to determine to what extent the severity of hypertension and the degree of blood pressure control contribute to the development of ESRD in negroes and mulattoes with glomerulonephritis. [Rev Assoc Med Bras 2002; 48(2): 167-7I]

KeY wORDS: Hypertension. End-Stage Renal Disease. Glomerulonephritis. Race. Negroes. Mulattoes.

\section{REFERÊNCIAS}

I. Lopes AA, Silveira MA, Martinelli R, Rocha $H$. Associação entre Raça e Estágio Final Doença Renal Secundária a Glomerulonefrite: Influência do Tipo Histológico e da Presença de Hipertensão Arterial. Rev Ass Med Brasil 200 I ; 47:78-84.

2. Rostand SG, Brown G, Kirk KA, Rutsky EA, Dustan HP. Renal insufficiency in treated essential hypertension. N Engl J Med 1989; 320:684-8.

3. Lopes AA, Port FK, James SA, Agodoa L. The excess risk of treated end-stage renal disease in blacks in the United States. J Am Soc Nephrol 1993; 3: |96|-7|

4. Lopes AA, Hornbuckle K, James SA, Port FK. The joint effects of race and age on the risk of hypertensive end-stage renal disease. Am J Kidney Dis 1994; 4:554-60.

5. Lopes AA, Port FK. Differences in the patterns of age-specific black/white comparisons between end-stage renal disease attributed and not attributed to diabetes. Am J Kidney Dis 1995; 25:7|4-2I.

6. Sorof JM, Hawkins EP, Brewer ED, Boydstun II, Kale AS, Powell DR. Age and ethnicity affect the risk and outcome of focal segmental glomerulosclerosis. Pediatr Nephrol 1998; 12:764-8.

7. Silbiger SR, Neugarten J. The impact of gender on the progression of chronic renal disease. Am J Kidney Dis 1995; 25:5 I5-33.

8. Pereira LJC, Martinelli R, Rocha H. Associação entre a função renal e hipertensão arterial em glomerulonefrites primárias. J Bras Nefrol 1994; 16:37-41.

9. D'Amico G. Influence of clinical and histological features on actuarial renal survival in adult patients with idiopathic IgA nephropathy, membranous nephropathy, and membranoproliferative glomerulonephritis: survey of the recent literature. Am J Kidney Dis 1992; 20:315-23.

10. Lopes AA. Raça e Incidência de Doença Renal Terminal em Pacientes com Glomerulonefrites Primárias. Tese de Livre Docência. Departamento de Medicina: Universidade Federal da Bahia, Salvador, BA, 1997.
II. Task Force on Blood Pressure Control in Children. Report of the Second Task Force on Blood Pressure Control in Children. National Heart, Lung, and Blood Institute, Bethesda, Maryland. Pediatrics 1987; 79: I-25.

12. Norusis MJ. SPSS for Windows: Base system user's guide, release 6.0. Chicago, IL: SPPS Inc, 1993.

13. Norusis MJ. SPSS for Windows: Advanced Statistics, release 6.0. Chicago, IL: SPSS Inc, 1993.

14. Lopes AA. Raça e Hipertensão Arterial. HiperAtivo 1996; 3: 153-62.

15. Lopes AA. Hipertensão Arterial: Fatores Étnicos e Raciais. J Bras Nefrol 1999; 21:82-44.

16. Cosio FG, Dillon JJ, Falkenhain ME, et al. Racial differences in renal allograft survival: the role of systemic hypertension. Kidney Int 1995; 47: || 36-4|.

17. Suthanthiran M, Khanna A, Cukran D, et al. Transforming growth factor-beta I hyperexpression in African American end-stage renal disease patients. Kidney Int 1998; 53:639-44.

18. Suthanthiran M, Li B, Song JO, et al. Transforming growth factor-beta I hyperexpression in AfricanAmerican hypertensives: A novel mediator of hypertension and/or target organ damage. Proc Natl Acad Sci U S A 2000; 97:3479-84.

19. Dustan HP. Growth factors and racial differences in severity of hypertension and renal diseases. Lancet 1992; 339:1339-40.

20. Silveira MA, Lopes AA, Martinelli R, Noblat ACB, Rocha $H$. Diferenças na Prevalência de Hipertensão Arterial entre Pacientes com Esclerose Glomerular Focal e Glomerulonefrite Membranoproliferativa. VII Congresso da Sociedade Brasileira de Hipertensão. Goiânia-GO, 05-08 de agosto de 1998.

21. Korbet SM, Genchi RM, Borok RZ, Schwartz MM. The racial prevalence of glomerular lesions in nephrotic adults. Am J Kidney Dis 1996; 27:647-51

22. Pontier PJ, Patel TG. Racial differences in the prevalence and presentation of glomerular disease in adults. Clin Nephrol 1994; 42:79-84.

23. Lopes AA, Martinelli RP, Silveira MA, Rocha $H$. Diferenças raciais entre pacientes com Esclerose Glomerular Focal e Glomerulonefrite Membranoproliferativa residentes no estado da Bahia. Rev Ass Med Bras 1999; 45: I I 5-20.

24. Ruggenenti P, Perna A, Lesti $M$, et al. Pretreatment blood pressure reliably predicts progression of chronic nephropathies. Kidney Int 2000; 58:2093-101.

25. Hebert LA, Kusek JW, Greene T, et al. Effects of blood pressure control on progressive renal disease in blacks and whites. Modification of Diet in Renal Disease Study Group. Hypertension 1997; 30:428-35.

26. Agodoa L. African American Study of Kidney Disease and hypertension (AASK): clinical trial update. Ethn Dis 1998; 8:249-53.

Artigo recebido: 20/12/2000

Aceito para publicação: 06/09/200 I 